\title{
Design and assessment of a low-cost, electromyographically controlled, prosthetic hand
}

This article was published in the following Dove Press journal:

Medical Devices: Evidence and Research

27 June 2013

Number of times this article has been viewed

\author{
Massimo Polisiero' \\ Paolo Bifulco' \\ Annalisa Liccardo² \\ Mario Cesarelli' \\ Maria Romano' \\ Gaetano D Gargiulo3 \\ Alistair L McEwan ${ }^{3}$ \\ Massimo D'Apuzzo² \\ 'Department of Biomedical, Electronics \\ and Telecommunication Engineering, \\ ${ }^{2}$ Department of Electrical Engineering, \\ University Federico II of Naples, \\ Naples, Italy; ${ }^{3}$ School of Electrical \\ and Information Engineering, \\ The University of Sydney, \\ New South Wales, Australia
}

Correspondence: Mario Cesarelli Department of Biomedical, Electronics and Telecommunication Engineering, University Federico II of Naples,

Via Claudio, 2I, 80I 25 Napoli, Italy

Tel +390817683767

Fax +3908I 7685925

Email cesarell@unina.it
Abstract: The study reported here explored the design and realization of a low-cost, electromyographically controlled hand prosthesis for amputees living in developing countries. The developed prosthesis is composed of a light aluminum structure with opposing fingers connected to a DC motor that imparts only the movement of grasp. Problems associated with surface electromyographic signal acquisition and processing, motor control, and evaluation of grasp force were addressed, with the goal of minimizing cost and ensuring easy assembly. Simple analog front ends amplify and condition the electromyographic signals registered from two antagonist muscles by surface electrodes. Analog signals are sampled at $1 \mathrm{kHz}$ and processed by a microcontroller that drives the motor with a supply voltage proportional to the muscular contraction, performing the opening and closing of the opposing fingers. Reliable measurements of the level of muscle contractions were obtained by specific digital processing: real-time operators implementing the root mean square value, mean absolute value, standard deviation, and mean absolute differential value were compared in terms of efficiency to estimate the EMG envelope, computational load, and time delay. The mean absolute value operator was adopted at a time window of 64 milliseconds. A suitable calibration procedure was proposed to overcome problems associated with the wide variation of electromyograph amplitude and background noise depending on the specific patient's muscles selected. A pulse-width modulated signal drives the DC motor, allowing closing and opening of the prosthesis. The relationship between the motor-driver signal and the actual hand-grasp force developed by the prosthesis was measured using a hand-held grip dynamometer. The resulting force was proportional to current for moderate values of current and then saturated. The motor torque, and, in turn, the force elicited, can be measured by sensing the current absorbed by the motor. Therefore, the grasp force can be opportunely limited or controlled. The cost of the only electronic and mechanical components of the electromyographically controlled hand was about US\$50; other costs, such as the cost of labor to assemble the prosthesis and the production of adapters for patients, were not estimated.

Keywords: electromyography amplitude measurements, electromyograph-controlled hand prosthesis, grasp-force measurements, low-cost design

\section{Introduction}

Loss of one or more limbs severely restricts the operational capability of a patient and their autonomy. In developing countries, causes of amputation are various, although most are associated with trauma due to war and landmines (responsible for about 22,000 amputations each year ${ }^{1}$ ), industrial or environmental accidents, and lack of basic public health facilities. ${ }^{2}$ Prosthetic hands seek to replace some functions of the human hand and their design is becoming more complex as increasingly sophisticated 
actions of the hand are replicated. This is expensive, so such hands are not always affordable by patients living in developing countries. The design of low-cost prostheses ${ }^{3,4}$ should consider restriction to basic functions, local availability of components, robustness and durability, ease of assembly, repair and maintenance and, of course, limitation of the total cost. Currently, in developing countries, the cost of a prosthetic limb still varies between US\$125 and US $\$ 1,875,{ }^{5}$ an expense that, considering the fact that the prosthesis must be replaced about every 4 years, can hardly be justified, especially in the case of children. ${ }^{6}$

Prostheses can be cosmetic only, activated by a cable, myoelectrically controlled, or a hybrid of these. ${ }^{7}$ Usually, for low-cost prostheses, cable activation is preferred: a mechanical gripper is set in motion by the combined movements of the shoulders or movements of the head (eg, extension of the neck). However, these require the patient to wear complex and cumbersome harnesses and vigorous effort is required to transmit force to the prosthesis. In contrast, active prostheses make use of actuators (usually electromechanical) powered by external energy (eg, batteries). The most common are controlled by electromyographic (EMG) signals ${ }^{8,9}$ recorded by surface electrodes, which are generally placed on muscles of the residual part of the limb. Several solutions have already been presented in the literature (eg, Su et al, ${ }^{10}$ Momen et al, ${ }^{11} \mathrm{Li}$ et $\mathrm{al}^{12}$ ) and are available on the market. Some offer a significant recovery of the functionality by means of three-fingered hands ${ }^{13,14}$ and are capable of replicating most of the movements of the arm, wrist, and hand. However, they are expensive for patients in developing countries (eg, produced by two leading companies in the field of prosthetics, the cosmetic prosthesis SensorHand Speed by Otto Bock [Duderstadt, Germany] and the i-Limb ${ }^{\mathrm{TM}}$ by Touch Bionics [Mansfield, MA, USA], cost US\$15,000 and US\$23,000, respectively).

The aim of this study was to design and test a very lowcost, electromyographically controlled hand prosthesis. In particular, problems related to the acquisition of the EMG signals using minimal hardware (HW) components, the measurement of muscular activity, the control of the electrical motor, and the characterization of the mechanical grip force exerted were addressed.

\section{Components of the prosthesis Design considerations}

To be accessible to as many amputees in developing countries as possible, the main concerns of the design were to reduce the cost of the components of the prosthesis and to provide a simple solution. As we considered that the building, assembling, and maintaining of the prosthesis should be done in the developing country in basic laboratories, ease of assembly and availability of components and materials were taken into account. These constraints excluded the vast majority of high-performance but complex solutions (eg, as in Micera et $\mathrm{al}^{15}$ ), which usually also involve feedback transducers. ${ }^{16}$

To allow the prosthesis to be used by children and people with limited strength and not encumber patients with burdensome mechanical apparatus like those activated by cables, an electromyographically controlled motorized solution was chosen. Further, the grasping force of the prosthesis was made proportional to the level of muscle contraction to provide intuitive usage and to favor a modulation of the movements by the patient, as is allowed in far more sophisticated prostheses.

\section{Mechanical parts}

The mechanical part of the prosthesis (Figure 1) is composed of a light aluminum structure and acts as a clamp (a thumb-like appendage and two opposing fingers) powered by a DC motor that imparts the only movement of grasp (one degree of freedom). Its total weight (motor included) is $230 \mathrm{~g}$.

The DC motor includes a linear actuator (MiniLAT 1.5A, Promoco Scandinavia, Taby, Sweden) and it can exert a maximum force of $150 \mathrm{~N}$ (about $15 \mathrm{kgf}$ ), at speed (at rated load) of $15 \mathrm{~mm} / \mathrm{s}$. Its nominal supply voltage is $12 \mathrm{~V}$ and it absorbs a maximum current of $1.5 \mathrm{~A}$. When no current is supplied to the motor, the linear actuator and gears are designed to retain the position of the fingers: to maintain a hold, no effort is required and muscle contraction is only used to vary the opening or closing of the clamp.

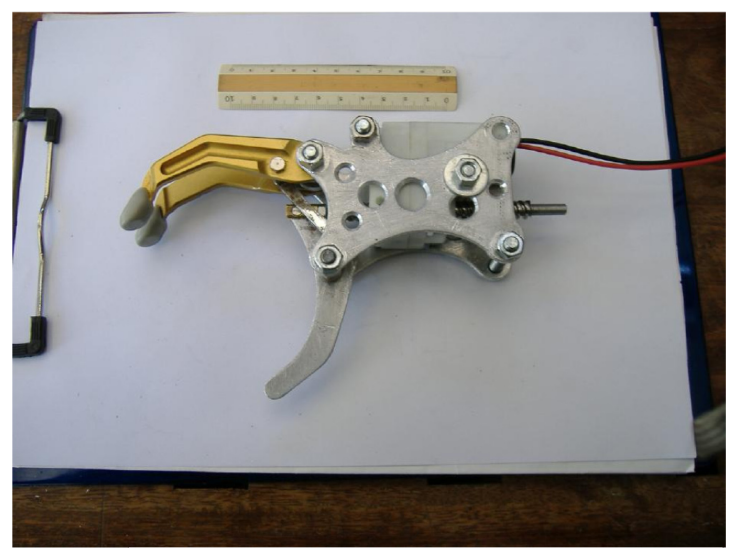

Figure I Mechanical structure of the prosthetic hand. Note: A $10 \mathrm{~cm}$ ruler is shown as a reference. 


\section{EMG front end}

Surface electromyography signals can be acquired from any two distinct muscles. In general, for transradial amputation, the remaining forearm muscles are considered. In particular, the flexor carpi ulnaris and extensor carpi radialis that act in wrist flexion and extension can be considered to drive the closing and the opening of the clamp, respectively. However, there is no particular limitation in the choice of the muscles (eg, two muscles of the shoulder involved in scapular rotation, etc). Electrical signals are acquired from the skin's surface by means of electrodes - in this case standard, disposable Ag/ $\mathrm{AgCl}$ electrodes (eg, which are currently employed in clinical electrocardiography) were used. The surface myoelectric potentials are very low in amplitude (ie, $20 \mu \mathrm{V}$ to $2 \mathrm{mV}$ ) and quite variable depending on many factors (eg, size of muscle fiber; depth, thickness, and composition of subcutaneous layers; electrode size; and inter-electrode distance).

Analog front ends were designed while keeping in mind the need to minimize the number of components and considering the cost and availability of electronic parts. Each channel consists of a MicroPower instrumentation amplifier (eg, half an INA2126; Burr-Brown Products from Texas Instruments, Dallas, TX, USA) that picks up EMG signals directly from the electrodes (Figure 2).

The differential amplifier (providing a common mode rejection ratio of $83 \mathrm{~dB}$ ) drastically reduces common mode signals such as power line interference and electrode polarization that can be two to three orders of magnitude higher than the EMG signal (which is usually lower than a few $\mathrm{mV}$ ). Gain at this stage was kept low (ie, $10 \mathrm{~V} / \mathrm{V}$ ) to accept large input variations without saturation. ${ }^{17,18}$ At the output of the instrumentation amplifier, was placed a first-order, passive high-pass filter (R1-C1, cutoff frequency of $400 \mathrm{~Hz}$ ) followed by a simple firstorder, active low-pass filter (R2-C2, cutoff frequency of $40 \mathrm{~Hz}$ )

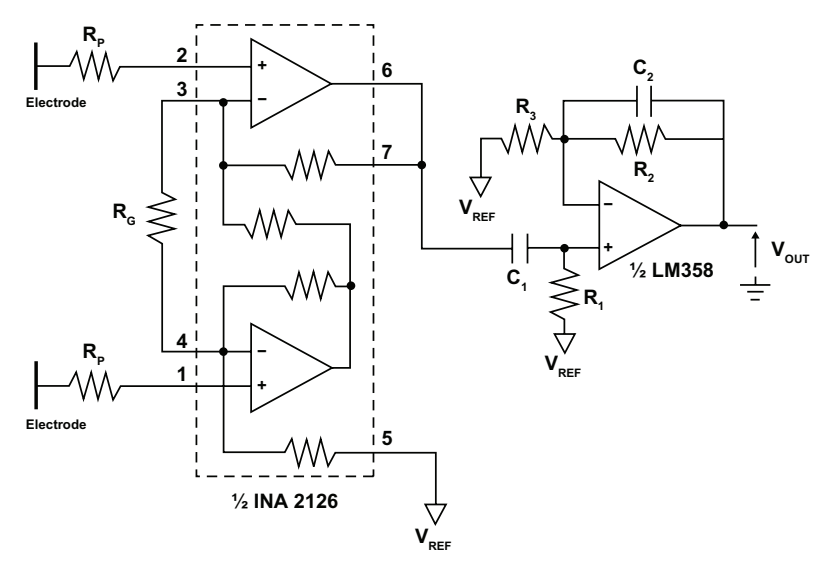

Figure 2 Front end of the electromyograph. realized by a single operational amplifier (ie, half an LM358 [Texas Instruments] $),{ }^{19}$ which provides a further amplification of $200 \mathrm{~V} / \mathrm{V}$ (R2/R3). A total gain of $2000 \mathrm{~V} / \mathrm{V}$ provides a suitable signal to be directly acquired by the analog to digital converter (ADC) of the microcontroller (5 V analog input range). Protection resistances $(\mathrm{Rp})$ were included in series to the electrode wires to comply with international standard IEC 60601-1 (Medical Electrical Equipment-Part 1: General Requirements for Basic Safety and Essential Performance). ${ }^{20}$

\section{Microcontroller and motor driver}

The processing unit of the system is based on a microcontroller-PIC18F23K22 by Microchip Technology (Chandler, AZ, USA) - characterized by very low power consumption (nanowatt technology), a 10-bit A/D converter, and a maximum operating speed of 16 MIPS. Figure 3 shows a block diagram of the control circuit of the prosthesis' motion. The microcontroller acquires the analog signals, performs signal processing, and controls the motor.

Each of the two EMG signals was band-limited to $400 \mathrm{~Hz}$ and a sampling frequency of $1 \mathrm{kHz}$ was adopted. Signals were quantized using a 10-bit ADC, providing a resolution of about $2.5 \mathrm{mV}$ for the raw EMG signals. Muscle activation levels were obtained by opportunely processing EMG samples in order to provide a unique motion command for the motor.

This information is coded as a pulse-width-modulated (PWM) signal by the microcontroller: the duty cycle of the PWM signal was made proportional to the measured EMG amplitude. The PWM signal is fed to a quadruple high-current half-H driver, which provides the desired bidirectional current to the DC motor. The integrated ST 293B by STMicroelectronics (Geneva, Switzerland) was selected. It is a quadruple high-current half-H driver characterized by a peak current of $2 \mathrm{~A}$. Each one of the $\mathrm{H}$ halfbridges is activated by an enable signal: the microcontroller generates the PWM signal and proper enabling signals to determine the motor's direction of rotation (ie, opening or closing of the prosthesis). During the generation of the PWM signal, the current absorbed by the motor is monitored. In particular, the microcontroller acquires, with a sampling frequency of $1 \mathrm{kHz}$, the voltage drop on a resistor of $5 \mathrm{~W}$ inserted into the supply circuit. The current absorbed by the motor provides information about the actual mechanical load of the prosthesis and can be used to limit or control the grasp force.

A single-plane printed circuit board was developed to host all the electronic components. Standard connectors allow wiring of the electrodes, the motor, and the battery. 


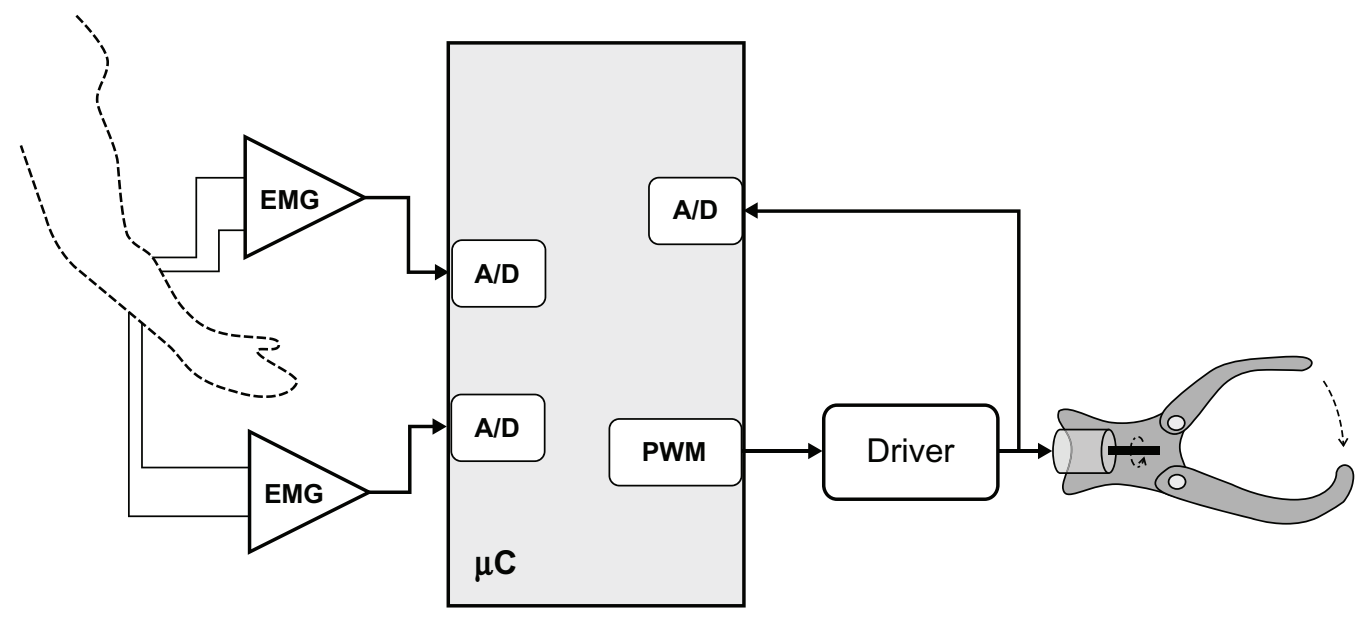

Figure 3 Block diagram of the control circuit of the prosthesis.

Abbreviations: A/D, analog to digital converter; EMG, electromyograph; PWM, pulse-width modulated.

When possible, passive components of equal value were chosen to reduce the total number of components required.

\section{EMG-signal amplitude measurement}

It is essential to provide real-time, reliable, and robust measurements of the muscular contractions.

The surface EMG signal is a stochastic signal formed by the interference of the motor unit's action potentials. The overall activity of a muscle and its exertion force is primarily related to the amplitude of this signal. The muscle activity intensity can be measured by computing the envelope - or, in general, the amplitude - of the surface EMG signal: the greater the local amplitude of the EMG, the more intense the muscle contraction.

For the surface EMG signal, various amplitude measurements were considered. ${ }^{21,22}$ One of the most widely used is the root mean square (RMS) amplitude, computed by calculating the RMS value for short successive periods (ie, a moving window) of the raw EMG signal. The mean absolute value (MAV) is a widely used EMG envelope estimator, which is based on full-wave rectification of the raw EMG signal and low-pass filtering. The local standard deviation (STD) and the mean absolute differential value (MDV) were included, while other more complex estimators $^{23}$ were not considered. All these measurements can be performed in real-time by considering a window of $\mathrm{N}$ samples that moves along the EMG signal. In summary:

$$
\mathrm{RMS}=\sqrt{\frac{1}{\mathrm{~N}} \sum_{\mathrm{n}=1}^{\mathrm{N}} \mathrm{x}_{\mathrm{n}}^{2}}
$$

$$
\begin{gathered}
\text { MAV }=\frac{1}{N} \sum_{n=1}^{N}\left|x_{n}\right| \\
\text { STD }=\sqrt{\frac{1}{N-1} \sum_{n=1}^{N}\left(x_{n}-\mu\right)^{2}} \\
\text { MDV }=\frac{1}{N} \sum_{n=1}^{N}\left|x_{n}-x_{n-1}\right|
\end{gathered}
$$

where " $\mathrm{x}_{\mathrm{n}}$ " is the measured EMG signal and " $\mathrm{m}$ " is the mean EMG over N samples.

All these measures of the level of muscle activity were compared for their suitability to control the prosthetic hand, taking into account the complexity of the calculations and promptness of the response to muscle action. Previous work ${ }^{24}$ showed that the widespread Gaussian model to represent the probability density function of the surface EMG signal does not exactly fit experimental EMG data, which are better represented by a Laplacian probability density function. For the Gaussian model, the local STD then the RMS are the best estimators of the EMG amplitude, while for the Laplacian model, the MAV operator provides a higher signal to noise ratio (SNR) than RMS processing. With reference to selection of the length of the time window, a shorter length provides a faster response to change in muscle activity (eg, onset of a contraction) but provides a more variable signal during steady effort. An optimal trade-off between the promptness and variability of the signal that controls the motor is desired.

Figure 4 represents the results provided by the different amplitude estimators by processing a raw EMG signal (sampled at $1 \mathrm{kHz}$ with a 10-bit ADC) recorded from a forearm muscle (top graph). Two distinct voluntary contractions appear evident (between seconds 1.0-2.0 and seconds 2.5- 

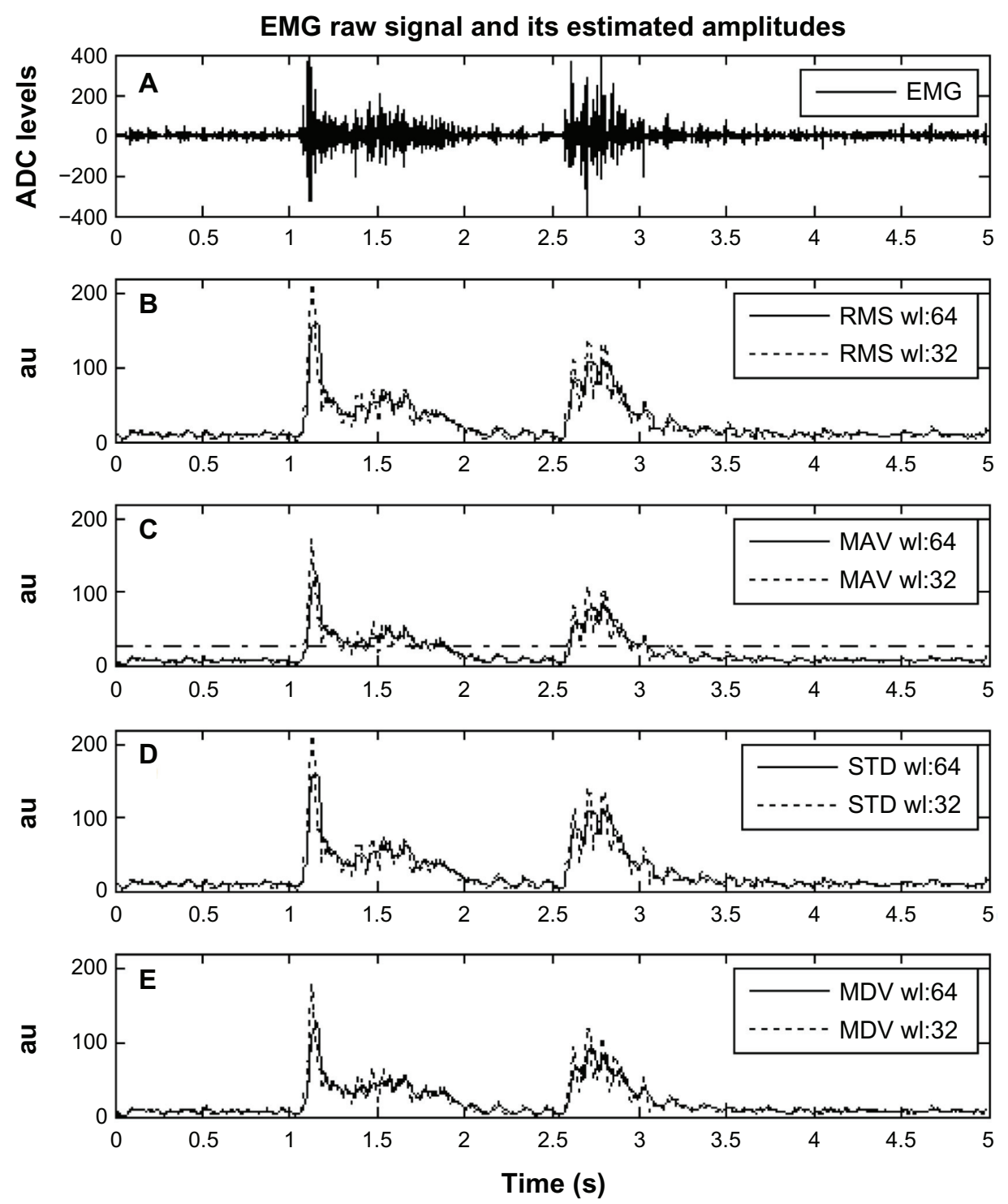

Figure 4 Example of raw electromyograph (EMG) (A) along with the real-time measurement of muscle activity as provided by the root mean square (RMS) (B), mean absolute value (MAV) (C), standard deviation (STD) (D), and mean absolute differential value (MDV) (E) operators.

Abbreviations: ADC, analog to digital converter; au, arbitrary units.

3.0), while the rest of the signal represents noise. Below, it is possible to appreciate on the same time axis the measurements of the muscle activity provided by the RMS, MAV, STD, and MDV operators. Effects of two different time window lengths are represented: a length of 32 milliseconds (dashed line) and 64 milliseconds (continuous line).

The cross-correlation index was computed for each pair of the RMS, MAV, STD, and MDV operators to quantitatively and concisely evaluate the similarity of the different measurements. For all the possible combinations of the two operators, the cross-correlation index was greater than 0.98 , indicating a substantial equivalence. Therefore, there was no particular reason for choosing one operator over another to control the electrical motor. With reference to the complexity of the operation to be performed by the microcontroller (see also Barzilay and $\mathrm{Wolf}^{25}$ ), there is no doubt that the MAV operator required the smallest complexity (by selecting windows length as power of two). Since the MAV operator was also preferable because it enhanced the SNR of the EMG amplitude estimation, ${ }^{24}$ it was finally chosen for our purpose. A window length of 64 milliseconds provided acceptably fast and accurate control of the motor. This window length is shorter compared with those suggested in other studies, ${ }^{26,27}$ but because of the simplicity of this solution (ie, as the control results were less ambiguous), a faster response was preferred.

\section{Motor control}

Once the envelope of the EMG signal from the two controllingmuscles was computed, it was necessary to implement a simple 
decision-making system for controlling the opening and closing of the prosthesis in a safe and non-ambiguous way.

The amplitude of the EMG signal is highly variable and depends on patient's anatomical conformation, properties of tissues, and electrode placement, among other factors: therefore, there is no standard placement and an individual customer setting was considered unavoidable. This is especially true for patients with amputations: the choice of electrode sites depends on the level of amputation and other peculiar factors; for practical reasons, the choice of the muscles may be modified in some scenarios.

A calibration procedure was developed to adapt the system functioning to different EMG amplitudes (eg montage from different muscles, different electrodes). The calibration procedure should be performed at the very moment of the application of the prosthesis to the patient (or whenever it is deemed appropriate). The electronic circuit was enriched with a few switches and light-emitting diodes to perform the calibration procedure: signals from switches create interruptions to the microcontroller (swapping to the calibration mode) and the light-emitting diodes provide intuitive visual feedback to the operators. The EMG signal is acquired for a short time (eg, about 1 second) both in the condition of rest and in the condition of maximum muscle contraction. This is done for each of the two muscles. When in resting condition, the average of the MAV values computed during the test is taken to be a noise floor value. Similarly, the maximum EMG amplitude is measured at the maximum voluntary contraction. Therefore, a threshold of muscle activation ("activity threshold") is defined: only when the EMG envelope exceeds this threshold, is the muscle considered voluntarily activated by the patient. The activity threshold can be chosen at two or three times the noise floor value. This reduces the sensitivity to small variations of EMG signal (eg, basal involuntary activity) and noise. See Figure 4C and consider an activity threshold set at 25 arbitrary units (dash-dotted horizontal line) to observe the periods of activation of the DC motor.

Further, only one muscle at time should be active to avoid ambiguity in motor control: a simultaneous activation of the opening muscle and the closing muscle must be ignored. Table 1 summarizes the operation of the decision-making process. When the decision to open or close the hand is made, the motor rotates with a torque proportional to the amplitude of the EMG.

\section{Electromechanical characterization}

Laboratory tests were carried out to experimentally evaluate the characteristic relationship between the current absorbed
Table I Criteria for the activation of the motor

\begin{tabular}{lll}
\hline & $\begin{array}{l}\text { Opening muscle } \\
<\text { activity threshold }\end{array}$ & $\begin{array}{l}\text { Opening muscle } \\
\text { >activity threshold }\end{array}$ \\
\hline $\begin{array}{l}\text { Closing muscle } \\
\text { <activity threshold }\end{array}$ & No action & OPEN hand \\
$\begin{array}{l}\text { Closing muscle } \\
\text { >activity threshold }\end{array}$ & CLOSE hand & No action \\
\hline
\end{tabular}

by the motor and the grasp force elicited by the prosthesis. Although the characteristics of the DC motor were known, this did not include the effect of the gears or of the joints included in the mechanics of the prosthesis. Essentially, the overall grasping force, as that of a human hand, is the actual output of the prosthesis. Therefore, an experimental measurement of the grasping force of the whole apparatus as a function of the current absorbed from the battery was made.

Knowledge of this relationship can help in enhancing the control of the motor and in predicting effects of battery discharge. In particular, the microcontroller, by measuring the current absorbed by the motor and by knowing the relationship current force of the prosthesis, can evaluate the actual reaction opposed from an object grasped by the hand: this feedback information can be used to adapt the behavior of the prosthesis. For example, the instant contact with an object is detected, the maximum force exerted can be limited or, when tightening around an object, the force can be opportunely modulated. This smart control can support the visual feedback of the patient.

Figure 5 shows the setup used to obtain the measurements. To resemble practical situation, a hand-held grip dynamometer (MAP 1.1; Kern and Sohn, Balingen, Germany), commonly used to evaluate the performance of subjects in clinical environments, was selected to measure the grasp. The dynamometer was set to allow a measurement range of 0-20 kgf with a resolution of $0.1 \mathrm{kgf}$. A specific mechanical

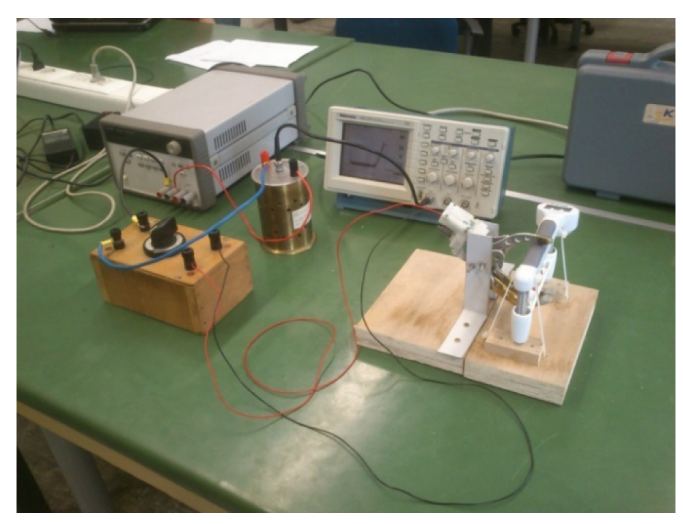

Figure 5 Measurement setup for prosthesis characterization. 
support was made to stabilize the relative positioning of the fingers of the prosthesis with respect to the dynamometer. The experimental setup also included: an Agilent Technologies E3648A power supply (Santa Clara, CA, USA) able to provide stabilized voltages from 0 to $20 \mathrm{~V}$ with a maximum current of $2.5 \mathrm{~A}$; a device to suitably reverse the current flow, in order to change the direction of the hand movement; a reference resistor, for gathering a voltage signal associated with the absorbed current, whose value of $0.5 \mathrm{~W}$ was chosen as a trade-off between the need to not interfere with the power supply circuit, and the need to ensure a proper amplitude of the voltage; and a Tektronix TDS 220 digital oscilloscope (Beaverton, OR, USA) for the acquisition of the voltage drop on the reference resistor.

The measurements were performed with three different supply voltages: $12 \mathrm{~V}, 10 \mathrm{~V}$, and $8 \mathrm{~V}$. By varying the current, the corresponding grasp force was registered by the dynamometer. Figure 6 summarizes the experimental relationships between current and grasp force that were obtained. As expected, the grasp force was found to be proportional to the current for low or moderate values of the current (fitting the linear relationships represented by dashed lines). Saturation of the grasp force is observable as the current reaches higher values. This appears more evident when the supply voltage decreases to $10 \mathrm{~V}$ and $8 \mathrm{~V}$.

\section{Conclusion and further directions}

A simple, low-cost, electromyographically controlled hand prosthesis intended for developing countries has been presented in this article. The HW and software presented are intended to be public and free. The design consid-

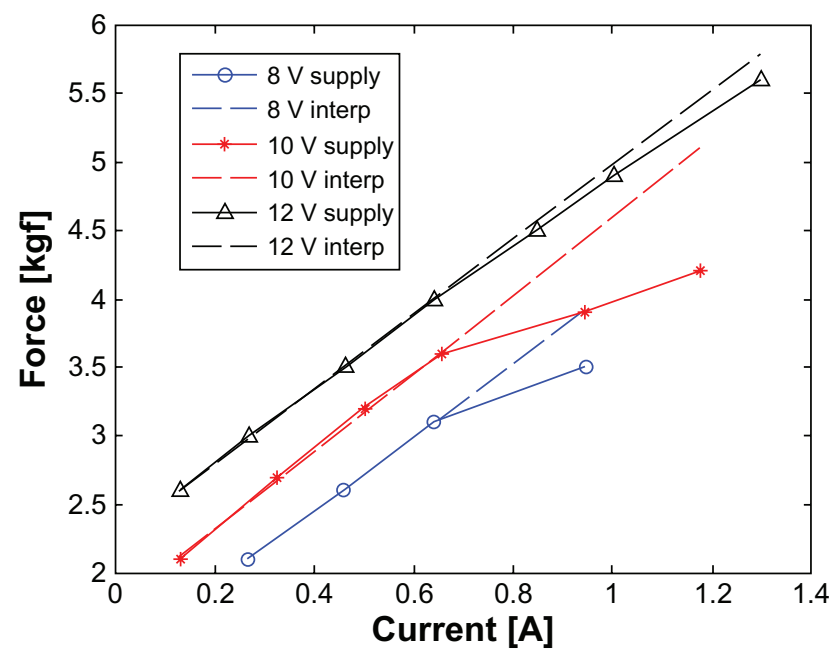

Figure 6 Measured grasp force versus absorbed current at different supply voltages. Abbreviation: interp, interpolation. ered a trade-off between reduced cost and technological performance. The cost of the electronic and mechanical components forming the prosthesis was estimated to be US\$50, with electronic components costing about US\$20 and the mechanical parts costing about US\$30 (US\$23 for the DC motor, with most of the remainder for the aluminum). Other costs, such as labor for assembling parts, the production of adapters for patients, training, and maintenance, were not estimated: these costs depend on the particular laboratory in the particular developing country. A standard small $12 \mathrm{~V}$ lead-acid battery (not included in costs) can supply the prosthesis.

The proposed prosthesis would be suitable for children, elderly, and weaker subjects, while for healthy and strong adults, entirely mechanical and body powered prostheses are likely to be more appropriate.

Some limitations are inherently associated with electromyographically controlled prostheses and to the presented solution. For example, the use of $\mathrm{Ag} / \mathrm{AgCl}$ disposable electrodes may pose a problem for long-term usage; as an alternative, stainless steel (or conductive rubber) dry electrodes are being tested (an elastic band wrapped around the forearm can be used to ensure a stable and durable contact between the electrodes and patient's skin).

Future studies will also focus on improvements in the control of the motor to facilitate specific operations and more detailed research will be directed toward evaluating the various types of noise ${ }^{28,29}$ that might hinder or degrade the correct functioning of the prosthesis. Most improvements will be achieved by changing the software of the microcontroller, not the structure of the HW. A further analysis of the performance will be performed on data collection and receipt of feedback from real patients.

\section{Acknowledgments}

The authors would like to thank M Serrafica and G Vigo for the practical realization of the mechanical parts of the prosthesis. Thanks also go to the nonprofit associations Time for Peace, Genova, and Engineering without Borders, Napoli, for supporting this project.

\section{Disclosure}

The authors declare no conflicts of interest in this work.

\section{References}

1. Berry D. From land mines to lawn mowers: prosthetic rehabilitation proceeds one foot at a time. The Washington Diplomat. Jan 2003

2. Engstrom B, Van de Ven C. Therapy for Amputees. 3rd ed. Edinburgh: Churchill Livingstone; 1999. 
3. Poonekar P. Prosthetics and orthotics in India. In: National Institute of Child Health and Human Development, National Institutes of Health. Prosthetic and Orthotic Research for the Twenty-first Century: Report of a Research Planning Conference: July 23-25, 1992, Bethesda, Maryland. Bethesda, MD: National Institute of Child Health and Human Development, National Institutes of Health; 1992:233-239.

4. Cummings D. Prosthetics in the developing world: a review of the literature. Prosthet Orthot Int. 1996;20(1):51-60.

5. Coupland RM. Assistance for Victims of Anti-Personnel Mines: Needs, Constraints and Strategy. Geneva: Geneva International Committee of the Red Cross; August 1997. Available from: http://www.markusthonius. de/downloads/mcoupland.pdf. Accessed May 20, 2013.

6. Walsh NE, Walsh WS. Rehabilitation of landmine victims - the ultimate challenge. Bull World Health Organ. 2003;81(9):665-670.

7. Micera S, Carpaneto J, Raspopovic S. Control of hand prostheses using peripheral information. IEEE Rev Biomed Eng. 2010;3:48-68.

8. Ha KH, Varol HA, Goldfarb M. Volitional control of a prosthetic knee using surface electromyography. IEEE Trans Biomed Eng. 2011;58(1): 144-151.

9. Jiang N, Englehart KB, Parker PA. Extracting simultaneous and proportional neural control information for multiple-DOF prostheses from the surface electromyographic signal. IEEE Trans Biomed Eng. 2009;56(4):1070-1080.

10. Su Y, Fisher MH, Wolczowski A, Bell GD, Burn DJ, Gao RX. Towards an EMG-controlled prosthetic hand using a 3-d electromagnetic positioning system. IEEE Trans Instrum Meas. 2007;56(1):178-186.

11. Momen K, Krishnan S, Chau T. Real-time classification of forearm electromyographic signals corresponding to user-selected intentional movements for multifunction prosthesis control. IEEE Trans Neural Syst Rehabil Eng. 2007;15(4):535-542.

12. Li G, Schultz AE, Kuiken TA. Quantifying pattern recognition-based myoelectric control of multifunctional transradial prostheses. IEEE Trans Neural Syst Rehabil Eng. 2010;18(2):185-192.

13. Carrozza MC, Dario P, Vecchi F, Roccella S, Zecca M, Sebastiani E. The CyberHand: on the design of a cybernetic prosthetic hand intended to be interfaced to the peripheral nervous system. Rep U S. 2003;3: 2642-2647.

14. Jingdong Zhao, Li Jiang, Shicai Shi, Hegao Cai, Hong Liu, Gerd Hirzinger.A five-fingered underactuated prosthetic hand system. In: Proceedings of the 2006 IEEE International Conference on Mechatronics and Automation, June 25-28, 2006, Luoyang, China. New York, NY: Institute of Electrical and Electronics Engineers; 2006:1453-1458.
15. Micera S, Rossini PM, Rigosa J, et al. Decoding of grasping information from neural signals recorded using peripheral intrafascicular interfaces. J Neuroeng Rehabil. 2011;8:53.

16. Cipriani C, Zaccone F, Micera S, Carrozza MC. On the shared control of an EMG-controlled prosthetic hand: analysis of user-prosthesis interaction. IEEE Trans Robot. 2008;24(1):170-184.

17. Gargiulo G, Calvo RA, Bifulco $P$, et al. A new EEG recording system for passive dry electrodes. Clin Neurophysiol. 2010;121(5):686-693.

18. Gargiulo G, Bifulco P, Cesarelli M, et al. An ultra-high input impedance ECG amplifier for long-term monitoring of athletes. Med Devices (Auckl). 2010;3:1-9.

19. Gargiulo G, Bifulco P, McEwan A, et al. Dry electrode Biopotential Recordings. Conf Proc IEEE Eng Med Biol Soc. 2010;2010: 6493-6496.

20. International Electrotechnical Commission (IEC). Medical Electrical Equipment - Part 1: General Requirements for Basic Safety and Essential Performance. Ed. 3.1. Technical standard IEC 60601-60601. Geneva: IEC; 2012.

21. Finsterer J. EMG-interference pattern analysis. J Electromyogr Kinesiol. 2001;11(4):231-246.

22. De Luca CJ. The use of surface electromyography in biomechanics. J Appl Biomech. 1997;13:135-163.

23. Cesarelli M, Bifulco P, Bracale M. Study of the control strategy of the quadriceps muscles in anterior knee pain. IEEE Trans Rehabil Eng. 2000;8(3):330-341.

24. Clancy EA, Hogan N. Probability density of the surface electromyogram and its relation to amplitude detectors. IEEE Trans Biomed Eng. 1999;46(6):730-739.

25. Barzilay O, Wolf A. A fast implementation for EMG signal linear envelope computation. J Electromyogr Kinesiol. 2011;21(4):678-682.

26. Smith LH, Hargrove LJ, Lock BA, Kuiken TA. Determining the optimal window length for pattern recognition-based myoelectric control: balancing the competing effects of classification error and controller delay. IEEE Trans Neural Syst Rehabil Eng. 2011;19(2):186-192.

27. Farrell TR, Weir RF. The optimal controller delay for myoelectric prostheses. IEEE Trans Neural Syst Rehabil Eng. 2007;15(1):111-118.

28. Fratini A, Cesarelli M, Bifulco P, Romano M. Relevance of motion artifact in electromyography recordings during vibration treatment. J Electromyogr Kinesiol. 2009;19(4):710-718.

29. Fratini A, La Gatta A, Bifulco P, Romano M, Cesarelli M. Muscle motion and EMG activity in vibration treatment. Med Eng Phys. 2009;31(9):1166-1172.
Medical Devices: Evidence and Research

\section{Publish your work in this journal}

Medical Devices: Evidence and Research is an international, peerreviewed, open access journal that focuses on the evidence, technology, research, and expert opinion supporting the use and application of medical devices in the diagnosis, treatment and management of clinical conditions and physiological processes. The identification of novel

\section{Dovepress}

devices and optimal use of existing devices which will lead to improved clinical outcomes and more effective patient management and safety is a key feature. The manuscript management system is completely online and includes a quick and fair peer-review system. Visit http://www. dovepress.com/testimonials.php to read real quotes from authors. 